\title{
Multiple Pontine Perforator Infarction in Basilar Atherosclerosis
}

\author{
Yong-Won Kim, Yang-Ha Hwang, Yong-Sun Kim
}

Keywords: Pontine infarction, basilar atherosclerosis, high-resolution MRI

doi:10.1017/cjn.2015.37

Can J Neurol Sci. 2015; 42: 264-266

\section{Case Presentation}

A-61-year-old man was admitted to our emergency department complaining of acute onset of dizziness, paresthesia, and numbness affecting the left hemibody and extremities without any motor symptoms. Electrocardiography showed a normal sinus rhythm without left ventricular hypertrophy. His medical history revealed only hypertension, and he was taking $100 \mathrm{mg}$ of losartan and aspirin. The initial neurological examination revealed decreased pain and temperature sensation in the left limb and hemibody without involvement of the face. Diffusionweighted imaging showed a high signal for the right dorsal tegmentum of the pons (Figure 1A). There was no apparent stenosis of the basilar artery on intracranial time-of-flight magnetic resonance angiography (TOF-MRA) (Figure 2A). After admission, his blood pressure ranged from 138/70 to $150 / 94$ $\mathrm{mmHg}$ after discontinuing a losartan. Three days after initial symptoms, he complained of newly developed slurred speech; follow-up diffusion-weighted imaging was taken, which revealed a new lesion in the right anterolateral pons and expansion of a right dorsal tegmental lesion (Figure 1B). Highresolution magnetic resonance imaging (HR-MRI) was performed for further angiographic evaluation, and we found a plaque surrounding an orifice of the branching arteries with strong contrast enhancement of the plaque (Figure 2B, top) in the mid-basilar artery (Figure 2B). HR-MRI was performed using a 3-T MRI (Signa Excite, GE healthcare, Milwaukee, WI) with an eight-channel head coil. Seven slices of contrastenhanced T1-weighted axial images were obtained with following parameters: repetition time/echo time $850 / 10 \mathrm{msec}$, field of view $12 \mathrm{~cm} \times 12 \mathrm{~cm}$, thickness $2 \mathrm{~mm}$, slice gap $0.3 \mathrm{~mm}$, matrix $320 \times 224$, and number of excitations 4 . Prolonged cardiac rhythm monitoring was also performed for three days and there was no evidence of atrial fibrillation.

Previous vascular and topographic studies of pontine infarctions revealed etiologies of vascular topography as follows: ${ }^{1-3}$
(1) anterolateral pontine infarction was usually caused by branch atheromatous disease of the basilar artery and (2) the tegmental pontine infarction usually arose from small artery disease. However, the main etiology of an unilateral multiple pontine infarction was not clear because of the paucity of case reports. ${ }^{2}$ The TOFMRA in this case showed no definitive stenosis from inherent limitations in showing the intraluminal pathology, but HR-MRI revealed an intraluminal atherosclerotic plaque with strong contrast enhancement (Figure 2B, top) in the right posterolateral aspect of the basilar artery and stenosis at the orifice of the branching arteries (Figure 2A-B). The strong contrast enhancement of the plaque was reported to be associated with vulnerable phenotype and inflammatory process. ${ }^{4,5}$ In this case, we demonstrated branch atheromatous disease as the etiology of the unilateral multiple pontine infarction and the role of HR-MRI in investigating the cause of the unilateral multiple pontine infarction.

\section{DISCLOSURES}

The authors do not have anything to disclose.

\section{REFERENCES}

1. Bassetti C, Bogousslavsky J, Barth A, Regli F. Isolated infarcts of the pons. Neurology. 1996;46:165-75.

2. Kumral E, Bayulkem G, Evyapan D. Clinical spectrum of pontine infarction. Clinical-MRI correlations. J Neurol. 2002;249: 1659-70.

3. Tatu L, Moulin T, Bogousslavsky J, Duvernoy H. Arterial territories of human brain: brainstem and cerebellum. Neurology. 1996; 47:1125-35.

4. Millon A, Boussel L, Brevet M, et al. Clinical and histological significance of gadolinium enhancement in carotid atherosclerotic plaque. Stroke. 2012;43:3023-8.

5. Millon A, Mathevet JL, Boussel L, et al. High-resolution magnetic resonance imaging of carotid atherosclerosis identifies vulnerable carotid plaques. J Vasc Surg. 2013;57:1046-51.

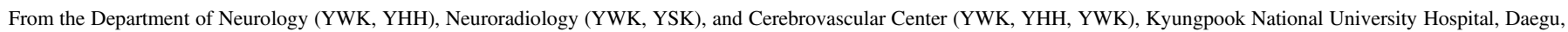
Republic of Korea School of Medicine, Kyungpook National University, Daegu, Republic of Korea. Received June 20, 2014. Final Revisions Submitted January 22, 2015.

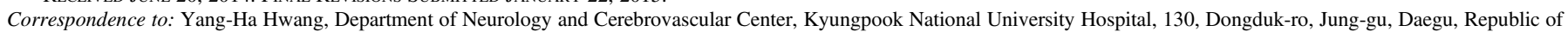
Korea 700-721. E-mail: yangha.hwang@gmail.com
} 

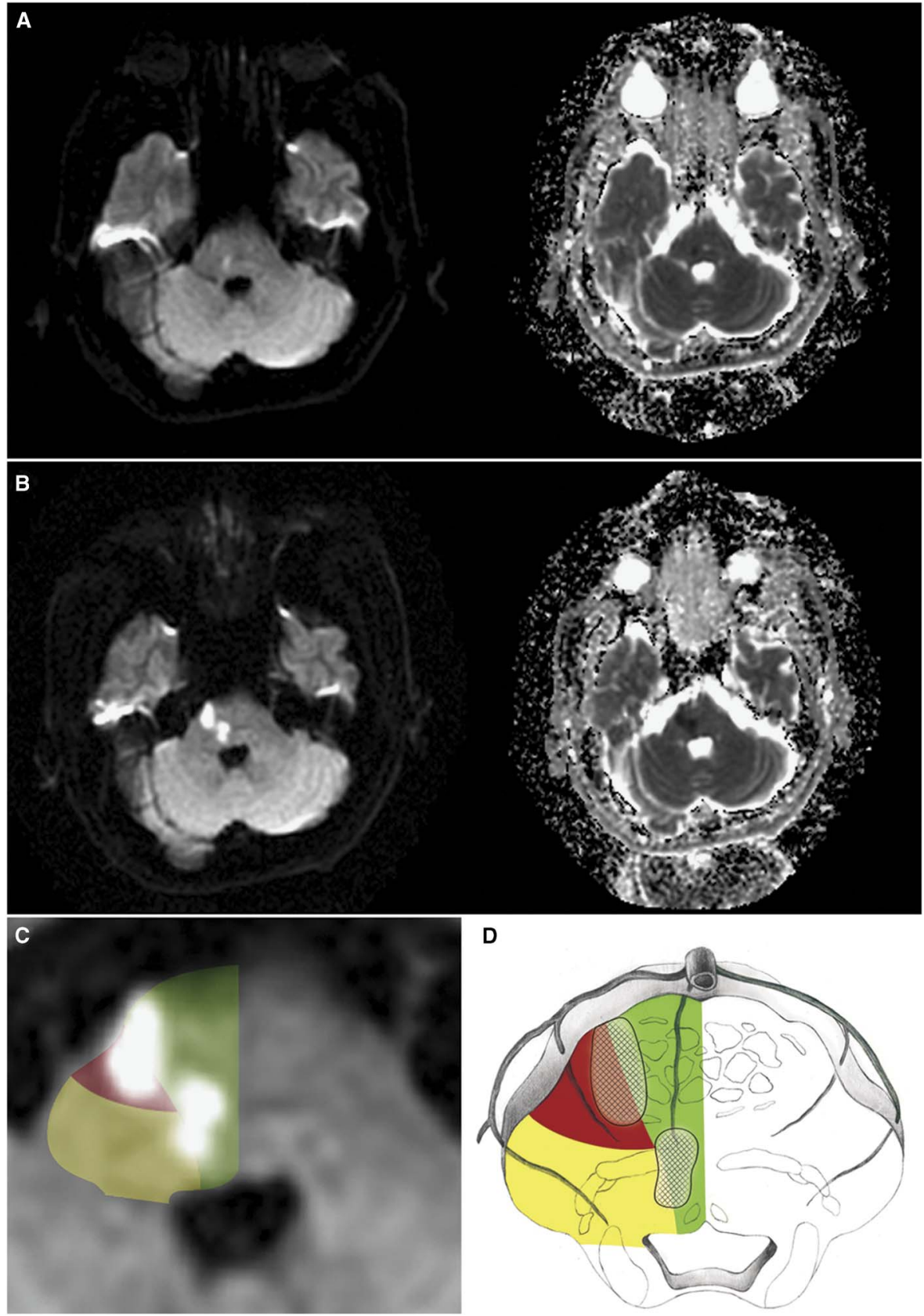

Figure 1: MRI of the patient. A, Baseline diffusion-weighted imaging and an apparent diffusion coefficient map showed an acute pontine infarction in the right tegmentum. B, Follow-up MRI showed an additional infarction in the right anterolateral pons. $C$ and D, Schema depicting the lesions according to vascular topography (green, anteromedial arterial territory; red, anterolateral arterial territory; yellow, lateral arterial territory). ${ }^{2}$ 


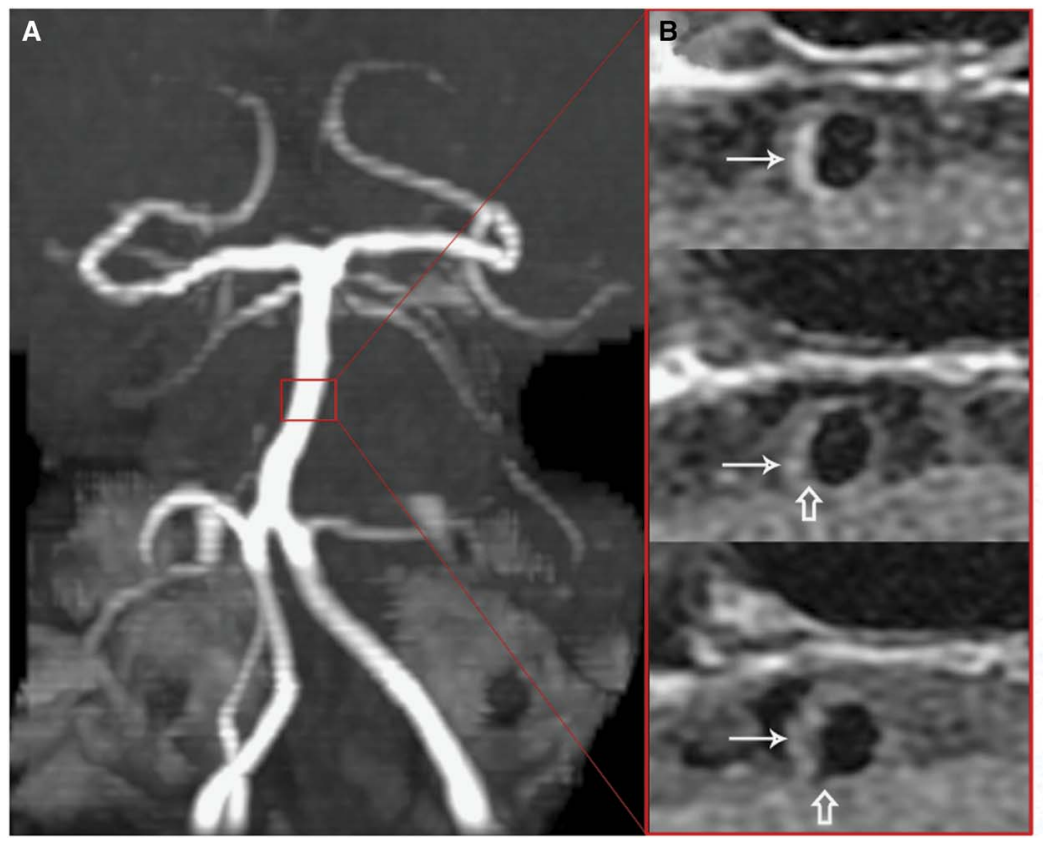

C

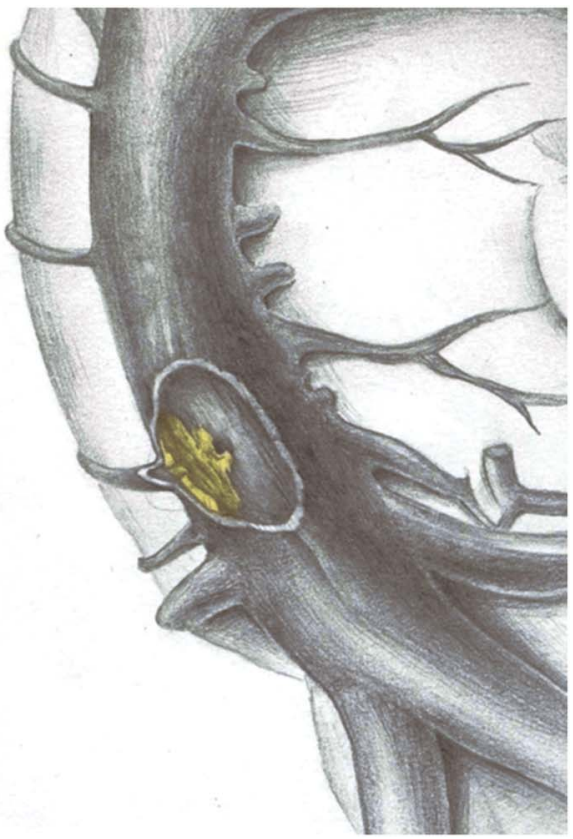

Figure 2: TOF-MRA and HR-MRI of the basilar artery. A, Normal appearance of the basilar artery in TOF-MRA. B, HR-MRI (contrastenhanced T1-weighted image) demonstrated an intraluminal atherosclerosis (thin arrow) with strong contrast enhancement (top) and stenosis of the orifice of the perforating arteries (thick arrow). C, Schema of atherosclerosis of the basilar artery. 